\title{
Performance of different methods for detecting T790M mutation in the plasma of patients with advanced NSCLC after developing resistance to first-generation EGFR-TKIs in a real-world clinical setting
}

\author{
TONGTONG HOU* ${ }^{*}$,JIAHAO ZENG ${ }^{*}$, HANYAN XU, SHANSHAN SU, JUNRU YE and YUPING LI \\ Department of Respiratory Medicine, The First Affiliated Hospital of Wenzhou Medical University, \\ Wenzhou, Zhejiang 325000, P.R. China
}

Received May 22, 2020; Accepted February 26, 2021

DOI: $10.3892 / \operatorname{mco} .2022 .2521$

\begin{abstract}
Several approaches to the detection of T790M mutations in patient plasma or tissue samples have been implemented to date. The present study was designed to assess the ability of different technologies to detect the T790M mutation in plasma samples and to evaluate the relative rates of re-biopsy and subsequent patient management in a clinical setting. Data from patients with advanced NSCLC who visited the Department of Respiratory Medicine of the First Hospital Affiliated to Wenzhou Medical University between December 2014 and July 2018 were retrospectively collected. Following re-biopsy, these patients were evaluated for the presence of the T790M mutation via next-generation sequencing (NGS), amplification refractory mutation system or Roche Cobas z480 (Cobas) analyses of tissue samples. T790M mutation status in tumor tissue samples was calculated as a standard reference used to establish the sensitivity, specificity and concordance of three circulating tumor DNA detection approaches, including NGS, droplet digital PCR (ddPCR) and super amplification refractory mutation system (SuperARMS). Subsequent patient management was also recorded. In total, 287 patients with advanced non-small cell lung cancer were evaluated, of whom $55.4 \%$ (159/287) underwent tissue re-biopsy, $76.7 \%(122 / 159)$ underwent sequencing analysis of plasma and/or tissue samples, and $59.0 \%(72 / 122)$ were found to harbor the T790M mutation. The rates of plasma sample T790M detection via
\end{abstract}

Correspondence to: Dr Yuping Li, Department of Respiratory Medicine, The First Affiliated Hospital of Wenzhou Medical University, Nanbaixiang Street, Wenzhou, Zhejiang 325000, P.R. China

E-mail:wzliyp@163.com

${ }^{*}$ Contributed equally

Key words: non-small cell lung cancer, epidermal growth factor receptor tyrosine kinase inhibitors, acquired resistance, T790M, re-biopsy
NGS, ddPCR and SuperARMS were 60.0, 59.3 and $60.0 \%$, respectively. Only 32 patients with T790M mutations (44.4\%, $32 / 72$ ) were treated with third-generation epidermal growth factor receptor tyrosine kinase inhibitors (EGFR-TKIs), while 19 continued treatment with first-generation TKIs, 13 underwent chemotherapy, 1 switched to treatment with anlotinib, 4 succumbed to pericardial or brain metastases, and 3 were lost to follow-up. Additionally, 2 patients exhibited histological transformation from adenocarcinoma to small cell lung cancer, while 17/97 patients who were evaluated for brain metastases during treatment exhibited intracranial progression. Of these, 8 patients had been treated with osimertinib. In this study of a real-world clinical setting, fewer patients than expected underwent re-biopsy and gene sequencing. Of the tools available for the analysis of plasma samples, NGS exhibited the highest sensitivity and concordance with the results of tissue-based T790M detection strategies. It was additionally found that only a subset of patients harboring the T790M mutation were ultimately treated using third-generation EGFR-TKIs.

\section{Introduction}

Lung cancer remains one of the most prevalent and deadly types of cancer globally (1), with the majority of patients with non-small cell lung cancer (NSCLC) first presenting with advanced or metastatic disease. Treatment planning in these patients is typically dependent upon tumor genomic profiling in order to identify the therapies to which a given patient is most likely to respond. Asian patients with NSCLC commonly harbor certain epidermal growth factor receptor (EGFR)-sensitizing mutations, such as L858R or exon 19 deletions $(2,3)$, and the treatment of these patients with EGFR tyrosine kinase inhibitors (EGFR-TKIs) has been shown to achieve significant improvements in progression-free survival (PFS) compared with first-line chemotherapy $(4,5)$. As such, EGFR-TKI monotherapy or combination treatment is a primary approach to the management of NSCLC patients with EGFR mutations (6). However, these patients generally develop acquired resistance to first-generation EGFR-TKIs over a median period of $10-14$ months $(7,8)$. 
Patients exhibiting progressive disease (PD) consistent with acquired resistance to first-generation EGFR-TKIs must typically undergo re-biopsy in order to establish the mechanism of resistance. The EGFR T790M mutation is the most common cause of such resistance and it is present in 50-60\% of the patients, with others exhibiting resistance as a result of either histological/phenotypic transformation or the activation of alternative pathways (9-11).

There are several approaches to detecting gene mutations in patient tissue or plasma samples. For patients with primary NSCLC, detecting gene mutations in tumor tissue samples using an amplification refractory mutation system assay is the clinical gold standard (12). This assay is based upon a quantitative PCR approach, and utilizes specific probes to detect mutations in tissue samples containing as little as $1 \%$ mutated DNA on a background of $99 \%$ normal DNA (13). Importantly, this assay has been approved for clinical use in China by the China Federal Drug Administration. However, this approach may be inadequate for re-biopsy tissue samples due to their limited DNA content. In order to reduce the incidence of false-negative results, more sensitive gene detection methods have been developed. Next-generation sequencing (NGS), which is based on the massively parallel sequencing of millions of different DNA molecules, allows for the detection of multiple mutations across genes. By using focused gene panels and by narrowing the coverage on target genes, each read is sequenced thousands of times, ensuring a high degree of sensitivity (14). The NGS approach facilitates the detection of rare circulating tumor DNA (ctDNA) sequences in the blood or other bodily fluids, and represents a new gold standard approach to ctDNA analysis (15). Roche Cobas $\mathrm{z} 480$ (Cobas) is an allele-specific polymerase chain reaction assay that enables the detection of known common EGFR mutations. The validity of this approach has been confirmed using negative and positive controls, revealing a sensitivity of $0.1-0.5 \%$ (16).

In some cases, conducting tissue biopsy can be dangerous due to the condition of the patient or the location of the tumor. Liquid biopsy may serve as an alternative option in these patients, as EGFR mutation status can be assessed using ctDNA. In certain clinical trials, droplet digital PCR (ddPCR) has been adopted to compare the performance of EGFR testing in ctDNA vs. tumor tissue samples, achieving high sensitivity and specificity $(17,18)$. However, as this approach is targeted, it limits the potential for the simultaneous analysis of additional emergent mutations. The super amplification refractory mutation system (SuperARMS) assay is a novel modification of the ARMS assay wherein primers and reaction conditions have been optimized to improve sensitivity, achieving a detection limit of $0.2 \%(6)$

Osimertinib is a third-generation EGFR-TKI that has been shown to be effective against tumors harboring T790M mutations and to achieve adequate central nervous system (CNS) concentrations. The AURA3 study demonstrated that osimertinib treatment achieved a significantly longer median PFS duration (10.1 vs. 4.4 months; $\mathrm{P}<0.001)$ and a better objective response rate ( 71 vs. $31 \%$; $\mathrm{P}<0.001$ ) relative to platinum-based chemotherapy combined with pemetrexed (19). Patients with acquired T790M mutation may be treated with osimertinib. The treatment of patients with other drug-resistant mechanisms, including MET rearrangement, HER-2 mutation and pathological transformation, may be changed to other targeted drugs or chemotherapy. Consequently, re-biopsy is necessary. Acquired T790M mutation represents 50-60\% of all resistance mechanisms, and patients with acquired T790M mutation show a good therapeutic response after receiving osimertinib as subsequent therapy (19). However, in the real-world clinical setting, the number of patients who receive osimertinib is lower compared with the rate of acquired T790M mutations reported in the literature, which may be attributed to the gene detection technologies or the patients' financial status (20). A number of different approaches have been used to assess tumor tissue and plasma samples in order to establish the presence of the T790M mutation, but the relative sensitivity and reliability of these different methods remain to be firmly established. In addition, the data from real-world clinical settings regarding the frequency of acquired T790M positivity in patients and their subsequent treatment are limited. Furthermore, tissue re-biopsy is not feasible for all patients; therefore, the present study was focused on plasma re-biopsy. The aim of the present study was to assess the relative sensitivity and reliability of different technologies for the detection of T790M mutations in plasma samples from patients with NSCLC and to assess re-biopsy rates and subsequent treatment strategies in a real-world clinical setting.

\section{Materials and methods}

Patients. Relevant data for all patients eligible for inclusion in this study were retrospectively gathered from the electronic records of the Department of Respiratory Medicine of the First Hospital Affiliated to Wenzhou Medical University between December 2014 and July 2018. All the patients were diagnosed with advanced NSCLC as confirmed by both radiological and cytological/pathological evidence. In addition, all patients harbored classical EGFR mutations (exon 19 deletion or the L858R mutation in exon 21), and all patients exhibited PD while under treatment with first-generation EGFR-TKIs.

Acquired resistance to first-generation EGFR-TKIs was defined based on a study conducted by Jackman et al (20) as follows: Patients exhibited significant and sustained benefits upon initial treatment with first-generation EGFR-TKIs (>6 months), but experienced PD within the past 30 days despite sustained treatment. Patients were excluded from the present study if they underwent additional treatment prior to re-biopsy, if they had additional cancers, or if they harbored de novo T790M mutations. The baseline disease status in each patient was evaluated within 28 days of EGFR-TKI treatment initiation, and patients were re-evaluated every 8 weeks via chest high-resolution computed tomography (CT) and every 2-3 months via brain, adrenal gland, lymph node, bone and abdominal imaging.

The Response Evaluation Criteria in Solid Tumors (version 1.1) (22) were used to define tumor progression. PFS-1 was defined as the time from initiation of first-generation EGFR-TKI treatment to the first documentation of PD, while PFS-2 was defined as the time from the initiation of sequential therapy until the date of objective PD or death.

All patient demographic and clinical information was retrieved retrospectively and based on real-world evidence, 
including patient age, sex, smoking history, tumor histological findings, mutation detection strategy, EGFR-activating mutation status, type of EGFR-TKI treatment, CNS metastasis status and type of PD.

This study was approved by the Ethics of Committees of the First Hospital Affiliated to Wenzhou Medical University, and was conducted in accordance with the 1964 Helsinki Declaration and its later amendments or comparable ethical standards. Informed patient consent was waived due to the retrospective study design.

Re-biopsy procedure. Tissue re-biopsy was conducted in patients via CT-guided needle biopsy, transbronchial biopsy, malignant lymph node biopsy, thoracoscopy biopsy and cytology from malignant effusions, as appropriate. All patients provided informed consent before re-biopsy was conducted. When tissue re-biopsy failed or when the obtained sample was insufficient for gene sequencing, plasma samples were collected for ctDNA analyses with patient consent. A peripheral whole blood sample $(10 \mathrm{ml})$ from each patient was collected into EDTA tubes and was used for subsequent gene detection analyses.

Assessment of EGFR T790M mutation status. Tissue samples from all patients were subjected to histological review, and when sufficient tumor cells were present, DNA was extracted from formalin-fixed paraffin-embedded (FFPE) samples for molecular analyses. Five to ten 5- $\mu \mathrm{m}$ sections per tumor tissue sample were used for DNA extraction.

The ARMS gene detection strategy utilized a QIAamp DNA FFPE Tissue Kit (Qiagen GmbH) according to the manufacturer's instructions. The Illumina Hiseq NovaSeq6000 S2 (Geneseeq Technology Inc.) platform was used for NGS analysis, while a multiplex Taqman real-time PCR by Cobas z480 (Roche Molecular Systems, Inc.) was used for Cobas. These systems were used to detect the presence of the T790M mutation and for other genomic analyses assessing ERBB2, ALK, RET fusion and ROS1 fusion status.

When plasma re-biopsy was required, ctDNA was extracted from the blood by centrifuging samples within $1 \mathrm{~h}$ of collection at room temperature $(2,000 \mathrm{x}$ g for $10 \mathrm{~min})$, followed by an additional spin at $8,000 \mathrm{xg}$ for $10 \mathrm{~min}$ to isolate the plasma, which was then stored at $-80^{\circ} \mathrm{C}$ until ctDNA extraction. Plasma ctDNA was extracted from $4 \mathrm{ml}$ of plasma from each patient. The samples were analyzed using an ADx-SuperARMS EGFR mutation detection kit (Amoy Diagnostics Co., Ltd.), Bio-Rad QX200 Droplet Digital PCR (Bio-Rad Laboratories, Inc.) and an NGS platform (Illumina Hiseq NovaSeq6000 S2, Geneseeq Technology Inc.). Eluted DNA was immediately used to detect EGFR mutations. All kits and techniques were used based on the manufacturer's instructions.

When one or more tissue gene detection analyses indicated T790M positivity, the patient was considered to harbor the T790M mutation in the analyzed tissue; the same was also true for plasma ctDNA detection results.

Statistical analysis. SPSS v24.0 (IBM Corp.) was used for all statistical testing. Sensitivity was measured by assessing the frequency of concordant sample positivity as a fraction of total tissue sample positivity, whereas specificity was determined based on the frequency of concordant negative samples as a fraction of total negative tissue samples. Concordance rates were determined based upon the sum of positives and negatives in both sample types divided by the total number of matched samples. The concordance of T790M detection in tissue and plasma samples was evaluated via the McNemar $\chi^{2}$ test and using $\kappa$ values. All time-to-event outcomes were estimated using the Kaplan-Meier method and compared across groups with the log-rank test or the Cox proportional hazards model. A two-sided $\mathrm{P}<0.05$ was considered to indicate statistically significant differences.

\section{Results}

Patient characteristics. In total, 287 patients were eligible for inclusion in the present study, of whom 103 did not undergo re-biopsy, and 25 only underwent plasma-based ctDNA analysis. The 159 patients who underwent tissue re-biopsy were included in our final patient cohort. These patients had a median age of 63 years (range, $32-85$ years), $63.5 \%$ were female, $78.6 \%$ were never-smokers and the remaining $21.4 \%$ were current or former smokers. In-frame exon 19 EGFR deletions were identified in $56 \%$ of included patients, with the remaining 44\% exhibiting L858R mutations in EGFR exon 21. Pathological analyses confirmed that the type of tumor was adenocarcinoma in all the patients. The first-generation EGFR-TKIs used to treat these patients included gefitinib $(n=74)$, icotinib $(n=82)$ and erlotinib $(n=3)$. The median PFS of the patients was 10.7 months. In total, 194 specimens were obtained upon re-biopsy of these 159 patients. All patient characteristics are listed in Table I, while the primary biopsy and re-biopsy methods are shown in Table II. A flowchart of the study design is shown in Fig. 1.

Prevalence of the T790M detection. Tissue re-biopsy was conducted in 159 patients, of whom 26 did not undergo any genetic analyses due to financial limitations. In addition, sufficient tissue was not obtained for genetic analyses from 23 patients, while 19 patients reportedly underwent re-biopsy and genetic analyses, but had no available gene reports and were lost to follow-up. In addition, 37 of these patients declined to provide blood samples; finally, only 122 patients underwent gene detection analyses based upon tissue, blood, or tissue and blood samples. The details on gene detection methods are shown in Fig. 4.

The presence of the T790M mutation was detected upon tissue re-biopsy in 53.8\% (49/91) of the patients, and upon plasma biopsy analysis in 56.6\% (47/83) of the patients. The rates of T790M mutation positivity in tissue specimens were $57.8 \%(26 / 45), 60.0 \%(12 / 20)$ and $38.1 \%$ (16/42) when detection was conducted using NGS, Cobas and ARMS assays, respectively. For plasma specimens, the rates of T790M mutation positivity were $60.0 \%(12 / 20), 59.3 \%$ (32/54) and $60.0 \%$ (9/15) when detection was conducted using NGS, ddPCR and SuperARMS assays, respectively.

In the 52 patients who underwent paired tissue and plasma sample analyses, the rates of T790M mutation positivity were 61.5 and $65.4 \%$, respectively $(\mathrm{P}=0.065)$. The sensitivity and specificity of the three methods for plasma T790M status determination were next evaluated by comparing the rates 
Table I. Characteristics of enrolled patients.

\begin{tabular}{|c|c|}
\hline Characteristics & $\mathrm{N}(\%)$ \\
\hline Age (years), median (range) & $66(40-81)$ \\
\hline \multicolumn{2}{|l|}{ Sex } \\
\hline Male & $58(36.5)$ \\
\hline Female & $101(63.5)$ \\
\hline \multicolumn{2}{|l|}{ Smoking status } \\
\hline Never smokers & $125(78.6)$ \\
\hline Former and current smokers & $34(21.4)$ \\
\hline \multicolumn{2}{|l|}{ Pathology at diagnosis } \\
\hline Adenocarcinoma & $157(98.7)$ \\
\hline Poorly differentiated carcinoma & $1(0.6)$ \\
\hline Sarcomatous degeneration & $1(0.6)$ \\
\hline \multicolumn{2}{|l|}{ Baseline EGFR mutation } \\
\hline Exon 19 deletions & $89(56.0)$ \\
\hline Exon $21 \mathrm{~L} 858 \mathrm{R}$ & $70(44.0)$ \\
\hline \multicolumn{2}{|l|}{ Initial EGFR-TKI regimen } \\
\hline Gefitinib & $74(46.5)$ \\
\hline Icotinib & $82(51.6)$ \\
\hline Erlotinib & $3(1.9)$ \\
\hline PFS-1 (months) & 10.4 \\
\hline PFS-2 (months) & 8.7 \\
\hline \multicolumn{2}{|l|}{ Disease stage } \\
\hline$<$ IIIB & 20 \\
\hline IIIB or IV & 139 \\
\hline
\end{tabular}

PFS, progression-free survival; EGFR-TKIs, epidermal growth factor receptor tyrosine kinase inhibitors. PFS-1, time from initiation of treatment with first-generation epidermal growth factor receptor tyrosine kinase inhibitors to the first documentation of progressive disease; PFS-2, time from the initiation of sequential therapy until the date of objective disease progression or death.

of detection of this mutation in paired plasma and tissue re-biopsy samples, with tissue samples serving as a reference. The T790M status in tissue and plasma is shown in Table III.

An NGS approach was employed to analyze plasma ctDNA samples from 19 patients. Of these, 8 patients were found to be T790M-positive in both tissue and plasma analyses, while 5 were negative in both analyses. Of the remaining patients, 2 were found to be T790M-positive in tissue samples but not in plasma samples, whereas 4 were found to be T790M-positive in plasma samples but not in tissue samples. By comparing these results, it was determined that the NGS-based detection of the T790M mutation in patient plasma had sensitivity and specificity values of $80 \%(8 / 10)$ and $55.6 \%$ (5/9), respectively.

A ddPCR-based approach was used to evaluate plasma ctDNA samples from 32 patients. Of these, 15 were found to be positive for T790M mutations in both tissue and plasma samples, whereas 6 were negative in both types of samples. By contrast, 7 patients were found to be positive for T790M mutations in tissue samples but not in plasma samples, while the remaining 4 patients were positive for T790M mutations in plasma samples but not in tissue samples. By comparing these
Table II. Primary biopsy and re-biopsy methods.

\begin{tabular}{lcc}
\hline Procedures & $\begin{array}{c}\text { Primary } \\
\text { biopsy, } \mathrm{n}(\%) \\
(\mathrm{n}=159)\end{array}$ & $\begin{array}{c}\text { Re-biopsy, } \\
\mathrm{n}(\%) \\
(\mathrm{n}=194)\end{array}$ \\
\hline $\begin{array}{l}\text { Computed tomography-guided } \\
\text { lung needle biopsies }\end{array}$ & $53(33.3)$ & $70(36.1)$ \\
Transbronchial biopsy & $42(26.4)$ & $26(13.4)$ \\
Thoracentesis & $33(20.8)$ & $76(39.2)$ \\
Thoracoscopic biopsy & $13(8.2)$ & 0 \\
Lung resection & $11(6.9)$ & $1(0.5)$ \\
Lymph node biopsy & $6(3.8)$ & $11(5.7)$ \\
Metastatic lesion surgery & $1(0.6)$ & $1(0.5)$ \\
Pericardiocentesis & 0 & $3(1.5)$ \\
Abdominocentesis & 0 & $4(2.1)$ \\
Lumbar puncture & 0 & $1(0.5)$ \\
\hline
\end{tabular}

results, it was determined that the ddPCR-based detection of T790M mutations in patient plasma had sensitivity and specificity values of $68.2 \%(15 / 22)$ and $60.0 \%$ (6/10), respectively.

A SuperARMS-based approach was used to evaluate the plasma ctDNA samples of 13 patients. Of these, 6 were found to be positive for T790M mutations in both tissue and plasma samples, whereas 1 was found to be negative in both sample types. By contrast, 4 patients were found to be positive for T790M mutations in tissue samples but not in plasma samples, while the remaining 2 patients were positive for T790M mutations in plasma samples but not in tissue samples. By comparing these results, it was determined that the SuperARMS-based detection of the T790M mutations in patient plasma had sensitivity and specificity values of $60.0 \%$ $(6 / 10)$ and $33.3 \%(1 / 3)$, respectively.

In conclusion, the rates of T790M positivity associated with these three different approaches in tissue and plasma were $52.6 \%(10 / 19)$ vs. $63.2 \%(12 / 19 ; \mathrm{P}=0.687), 68.8 \%(22 / 32)$ vs. $59.4 \%(19 / 32 ; \mathrm{P}=0.549)$ and $76.9 \%(10 / 13)$ vs. $61.5 \%$ $(8 / 13 ; \mathrm{P}=0.687)$, respectively. Further details may be found in Table IV. The rates of T790M detection did not differ significantly between tissue and plasma samples $(\mathrm{P}>0.05)$, with a low concordance rate between the two $(\kappa<0.4)$, possibly due to the insufficient sample size included in this study.

Prevalence of other detected mutations. In total, 122 patients underwent T790M genotyping, of whom 72 were found to be positive for this mutation. Of these patients, 2 exhibited dual MET and EGFR T790M mutations, while 1 harbored dual ALK and EGFR T790M mutations.

The remaining 50 patients were T790M-negative. Of these patients, 4 had rare mutations, including ALK fusions as well as HER-2, EGFR G719A and EGFR G719X mutations.

Subsequent treatment. A total of $76.7 \%$ (122/159) of the patients in the present study underwent re-biopsy and/or plasma-based genotyping following tumor progression. Of these patients, 72 (59\%) were found to be T790M-positive, yet only 32 patients $(26.2 \%, 32 / 122)$ were treated with 


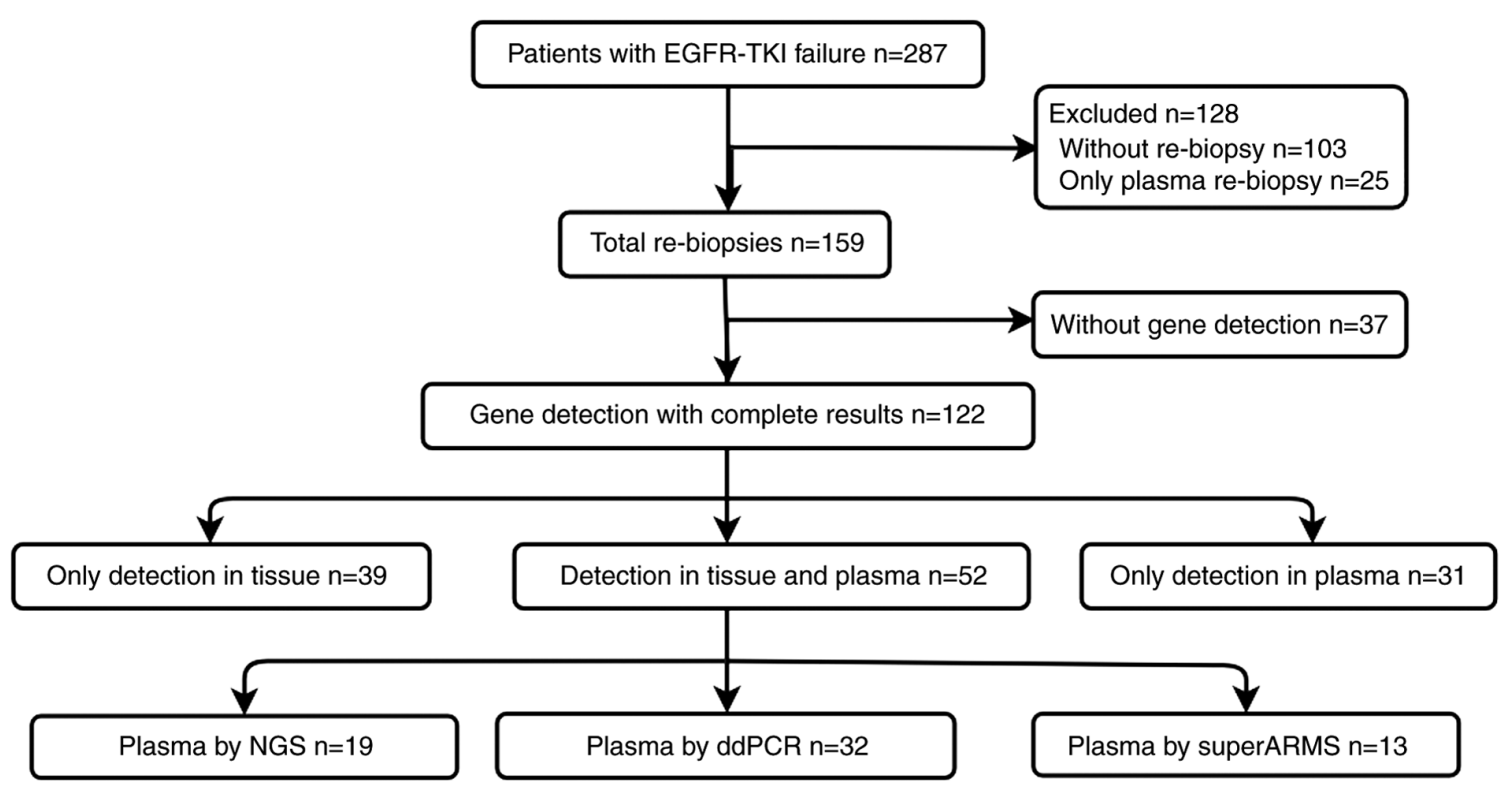

Figure 1. Flowchart of the study design. EGFR-TKI, epidermal growth factor receptor tyrosine kinase inhibitor; NGS, next-generation sequencing; ddPCR, droplet digital PCR; SuperARMS, super amplification refractory mutation system.

osimertinib. Of the remaining patients, 19 continued to receive first-generation EGFR-TKIs, 13 switched to a chemotherapy regimen, 1 switched to anlotinib, 4 died from pericardial or brain metastases, and 3 were lost to follow-up.

Association between the T790M mutation and survival in patients receiving osimertinib. PFS-1 was assessed in all 122 patients based on their tissue or plasma T790M status. In all patients, the median PFS-1 was 10.4 months. The median PFS-1 in the T790M-positive group was shorter compared with that in the T790M-negative group (10.3 vs. 10.7 months, respectively; $\mathrm{P}=0.752$; Fig. 2 ).

The survival of 64 patients with T790M mutations who underwent different sequential treatments, including osimertinib, chemotherapy and sustained first-generation TKI therapy, was also investigated (Fig. 3). The median PFS-2 in these patients was 8.7 months (95\% CI: 6.338-11.026 months). Of these patients, the median PFS-2 of 32 patients receiving osimertinib was 9.1 months (95\% CI: 8.012-10.118), the median PFS-2 of the 13 patients who switched to chemotherapy was 6.8 months (95\% CI: 3.484-10.116), and the median PFS-2 of the 19 patients who received continuous TKI treatment was 2.9 months (95\% CI: 2.740-3.060). Of these three groups, the PFS-2 of patients receiving osimertinib was significantly longer compared with that of patients who underwent themotherapy or sustained first-generation TKI treatment $(\mathrm{P}<0.001)$.

\section{Discussion}

EGFR mutations are among the most common driver mutations observed in Asian patients with NSCLC. First-generation EGFR-TKIs, such as gefitinib or erlotinib, or second-generation EGFR-TKIs, such as afatinib, remain the standard of care for patients with advanced NSCLC harboring EGFR-sensitizing mutations. While these EGFR-TKIs are associated with good efficacy and improved patient quality of life, drug resistance commonly develops and remains an obstacle to positive long-term treatment outcomes.

In patients exhibiting PD, re-biopsy is required in order to understand the genetic basis for resistance and to guide further treatment selection. Tissue re-biopsy samples exhibit higher rates of T790M mutation and are more stable compared with plasma samples. In addition, tissue samples enable detection of histological transformation. Therefore, tissue specimens are used for reference purposes upon re-biopsy (21). However, as tissue re-biopsy is an invasive procedure, it may not be well-tolerated by certain patients. In addition, it may be difficult to obtain such biopsy samples when patients exhibit stable lung disease but experience progression in other sites, such as the brain, lung, bone, or peritoneum. Liquid biopsy, by contrast, is a low-risk and relatively non-invasive procedure that can offer more comprehensive information regarding tumor mutation status without the spatial restrictions imposed by tissue re-biopsy. In theory, liquid biopsy can offer a comprehensive overview of patient tumor burden and mutational status, although in practice such biopsies generally only reflect a small fraction of total tumor DNA present within cancer patients (22). Indeed, in some cases, plasma ctDNA analyses may fail to detect mutations that are identified upon analysis of tumor tissue specimens. Therefore, negative plasma ctDNA results should be confirmed by tumor tissue sample analyses (23-25). Recent work by Usui et al (26) utilized a Cobas approach to detect the T790M mutation in plasma ctDNA re-biopsy samples, and determined that this approach had a sensitivity of $2 / 9(22.2 \%)$ and a specificity of $15 / 22(68.2 \%)$, with a T790M detection concordance rate of $54.8 \%$ between plasma and tissue samples. Zugazagoitia et al (27) concluded that NGS-based analyses of ctDNA may serve as an alternative to tumor tissue sample analyses in their small study of 53 patients. A study of 95 patients conducted by Cui et al (28) found that SuperARMS and ARMS methods exhibited high concordance in their detection of EGFR mutations. With respect to T790M mutation status, 9 cases were identified via 
Table III. T790M status in tissue and plasma.

Plasma T790M status, n (\%)

\begin{tabular}{|c|c|c|c|c|c|c|c|c|c|}
\hline \multirow{2}{*}{$\begin{array}{l}\text { Tissue } \\
\text { T790M } \\
\text { status }\end{array}$} & \multicolumn{3}{|c|}{ By NGS } & \multicolumn{3}{|c|}{ By ddPCR } & \multicolumn{3}{|c|}{ By SuperARMS } \\
\hline & + & - & Total & + & - & Total & + & - & Total \\
\hline+ & 8 & 2 & $10(52.6)$ & 15 & 7 & $22(68.8)$ & 6 & 4 & $10(76.9)$ \\
\hline- & 4 & 5 & $9(47.4)$ & 4 & 6 & $10(31.2)$ & 2 & 1 & $3(23.1)$ \\
\hline Total & $12(63.2)$ & $7(36.8)$ & $19(100.0)$ & $19(59.4)$ & $13(40.6)$ & $32(100.0)$ & $8(61.5)$ & $5(38.5)$ & $13(100.0)$ \\
\hline
\end{tabular}

NGS, next-generation sequencing; ddPCR, droplet digital PCR; SuperARMS, super amplification refractory mutation system.

Table IV. Sensitivity and specificity of different detection methods.

\begin{tabular}{|c|c|c|c|c|c|c|}
\hline \multirow[b]{2}{*}{ Detection method } & \multicolumn{2}{|c|}{ Detection rate $(\%)$} & \multirow[b]{2}{*}{ P-value } & \multirow[b]{2}{*}{ Sensitivity (\%) } & \multirow[b]{2}{*}{ Specificity (\%) } & \multirow[b]{2}{*}{$\kappa$} \\
\hline & Tissue & Plasma & & & & \\
\hline Plasma NGS & 52.6 & 63.2 & 0.687 & 80.0 & 55.6 & 0.360 \\
\hline Plasma ddPCR & 68.8 & 60.0 & 0.549 & 68.2 & 60.0 & 0.261 \\
\hline Plasma SuperARMS & 76.9 & 61.5 & 0.687 & 60.0 & 33.3 & -0.054 \\
\hline
\end{tabular}

NGS, next-generation sequencing; ddPCR, droplet digital PCR; SuperARMS, super amplification refractory mutation system.

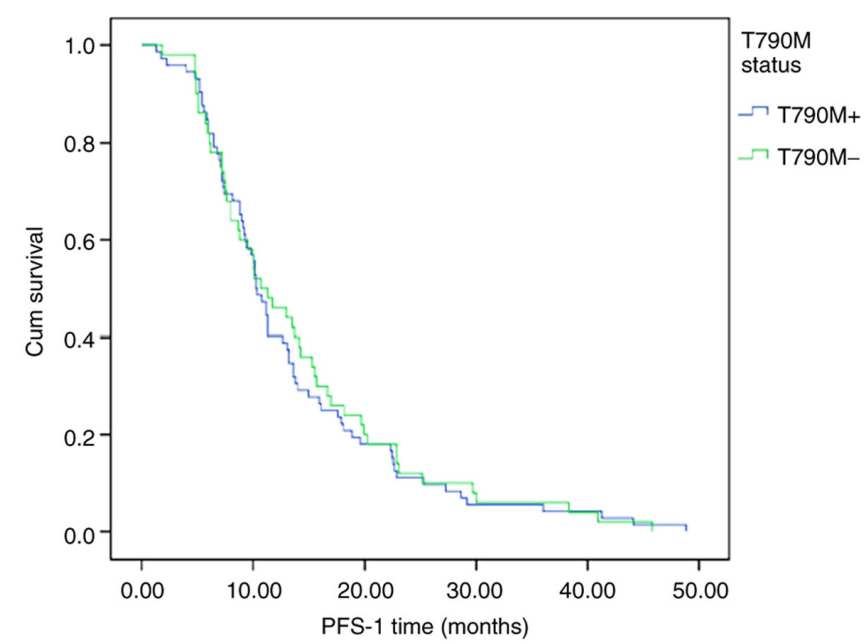

Figure 2. Kaplan-Meier curve analysis demonstrated that patients with and without T790M did not exhibit statistically significant differences in median PFS-1 ( $<<0.001)$. PFS, progression-free survival; PFS-1, time from initiation of treatment with first-generation epidermal growth factor receptor tyrosine kinase inhibitors to the first documentation of progressive disease.

SuperARMS, whereas only 1 of these cases was detected using an ARMS assay. However, at present, there is not sufficiently strong evidence to support the use of ctDNA-based analyses for the identification of the EGFR T790M mutation in patients with NSCLC.

In the present study, 52 patients who had undergone simultaneous analyses of tissue and plasma re-biopsy samples were identified, yielding respective rates of T790M positivity of 61.5 and $65.4 \%(\mathrm{P}=0.065)$. In contrast to prior studies, the

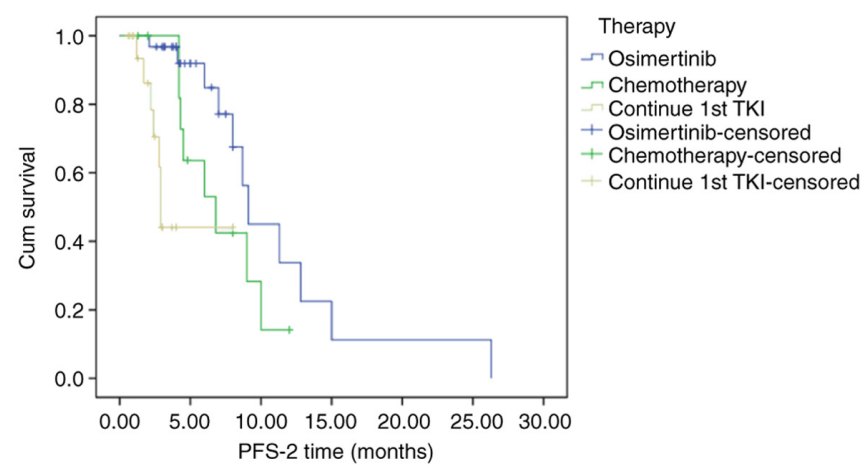

Figure 3. Kaplan-Meier curve analysis demonstrated that patients harboring T790M mutation treated with osimertinib had a longer PFS-2 compared with the subgroup treated with chemotherapy or first-generation TKIs $(\mathrm{P}<0.001)$. PFS, progression-free survival; PFS-2, time from the initiation of sequential therapy until the date of objective disease progression or death; TKIs, tyrosine kinase inhibitors.

rate of T790M detection herein was slightly lower in tissue samples. This may be attributable to the different methodological approaches used to analyze these samples, or it may be a consequence of tumor tissue heterogeneity and the presence of T790M subclones in plasma ctDNA samples $(29,30)$.

In the present study, the $\mathrm{T} 790 \mathrm{M}$ detection rates in tissue samples were 57.8 and $60.0 \%$ by NGS and Cobas, respectively, in line with the detection rates of $49-65 \%$ reported by prior studies $(19,31,32)$. A number of retrospective analyses have explored ARMS-based genotyping and found it to be modestly sensitive when detecting T790M mutations, with a recent study having recorded a rate of $\mathrm{T} 790 \mathrm{M}$ positivity of $39.6 \%$ using an 


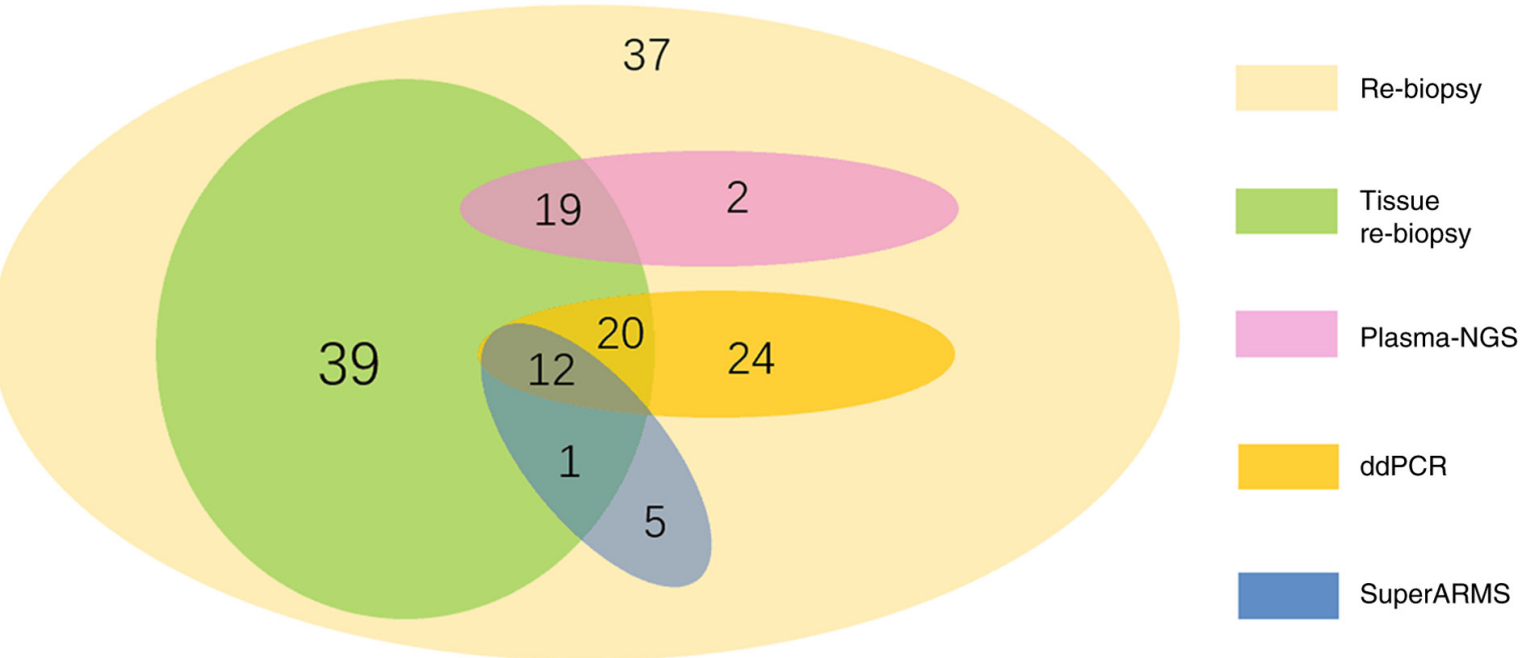

Figure 4. Distribution of the159 patients subjected to tissue and plasma re-biopsy. NGS, next-generation sequencing; ddPCR, droplet digital PCR; SuperARMS, super amplification refractory mutation system.

ARMS approach (33), similar to our findings. Relative to other tested genotyping strategies, ARMS is not as sensitive, particularly when detecting low-abundance mutations. While the detection of T790M and other EGFR mutations via ARMS has been increasingly common in the clinical setting, the relatively poor sensitivity of this assay bears the risk of erroneously low rates of accurate detection for this mutation in tissue samples.

To further evaluate the sensitivity and specificity of different ctDNA-based T790M mutation detection approaches, these 52 patients were next subdivided based on whether they were analyzed via NGS, ddPCR or SuperARMS. It was observed that NGS was the most sensitive of these approaches and exhibited moderate specificity, as well as the highest concordance with tissue testing among these three strategies. All three methods had sensitivity values of $60-80 \%$, in line with the ranges reported in prior studies (40-78\%). These three methods had specificities of 55.6-60\% (with the exception of SuperARMS, which had a specificity of just $33.3 \%$ due to an insufficient sample size and was thus omitted), with these values being well below those reported by prior studies $(80-100 \%)(22,34-36)$. This may be the result of the insufficient number of patients undergoing simultaneous blood- and tissue-based genotyping in the present study.

As the availability of osimertinib to patients within the Chinese healthcare system has increased in recent years, the proportion of patients undergoing re-biopsy has also increased. Specimen types and analysis methods have also become more diversified in recent years, leading to the more widespread use of third-generation EGFR-TKIs. However, our results suggest that only a limited subset of patients are actually treated with osimertinib as second-line therapy after developing resistance to first-generation EGFR-TKIs.

Of the 287 patients included in the present study, 128 did not undergo re-biopsy. For the majority of these patients, the decision not to perform re-biopsy was associated with either an intolerance to the necessary biopsy procedures, difficulty in accessing the biopsy site, or financial limitations. In total, 159 patients underwent tissue re-biopsy, but the sequencing of tissue samples from 68 of these patients failed due to insufficient tumor content $(n=25)$ or negative pathology $(n=12)$. In total, $122(76.7 \%, 122 / 159)$ patients underwent tissue and/or alternative plasma-based genotyping of T790M. Of these, 72 patients $(59 \%, 72 / 122)$ were found to be T790M-positive. Ultimately, only 32 patients $(26.2 \%$, $32 / 122$ ) were subsequently treated with osimertinib. Decisions regarding the use of osimertinib were primarily related to the general patient condition and financial factors.

These results clearly indicated that the percentage of patients treated with third-generation EGFR-TKIs was markedly lower than the rate at which this mutation was detected in this real-world clinical setting. A number of different factors ultimately determine whether patients are able to undergo re-biopsy and to be treated with osimertinib if appropriate, including limited financial means, difficulties in conducting re-biopsy due to patient intolerance or tumor location, and failed re-biopsy. To date, two real-world studies have confirmed that $<25 \%$ of patients exhibiting acquired resistance to first-generation EGFR-TKIs were able to undergo treatment with osimertinib $(37,38)$. The FLAURA study found that the use of osimertinib as first-line therapy in NSCLC patients with EGFR mutations was associated with a significant increase in median PFS relative to first-line use of first-generation EGFR-TKIs (18.9 vs. 10.2 months, respectively; $\mathrm{P}<0.0001$ ) (39). Therefore, the use of osimertinib as first-line therapy may be beneficial for patients with EGFR-mutated NSCLC.

Brain metastases are often detected in patients with advanced NSCLC, with $>25 \%$ of patients exhibiting such metastases upon initial diagnosis and with their prevalence increasing with disease progression. EGFR mutation positivity and EGFR-TKI treatment are associated with a higher incidence of brain metastases in patients with advanced NSCLC (40). The mechanism underlying this phenotype may be distinct from that governing extracranial progression. Specifically, while extracranial progression is often associated with acquired resistance, intracranial progression is generally associated with the limited ability of EGFR-TKIs to penetrate the blood-brain barrier (BBB). Indeed, gefitinib and erlotinib have just 1.1 and 2.8-5.1\% BBB penetration rates, respectively $(41,42)$. A total of 97 patients in the present study 
were evaluated for brain metastases during treatment. Of these, 20 patients exhibited brain metastases at baseline, with 11 exhibiting intracranial progression during treatment and 8 exhibiting intracranial stability $(8.2 \%, 8 / 97) ; 1$ patient with baseline lesions exhibited a partial response (1\%, 1/97). Of the 77 patients without baseline brain metastases prior to first-line EGFR-TKI treatment, 6 exhibited new intracranial metastases during follow-up. In summary, 17 patients in the present study exhibited intracranial progression, with 8 patients $(47.1 \%, 8 / 17)$ having been treated with osimertinib, 3 (17.6\%, 3/17) having been treated via chemotherapy, and 4 patients $(23.5 \%, 4 / 17)$ under continued treatment using first-generation EGFR-TKIs. In addition, 2 patients exhibited rapid intracranial progression and succumbed to the disease within 2 weeks.

A total of 2 patients in our overall study population (1.3\%, 2/159) who underwent re-biopsy exhibited a rare histological transformation from adenocarcinoma to small cell lung cancer (SCLC). As chemotherapy is the primary treatment used for SCLC, 1 of these patients received chemotherapy, although it was found to be ineffective after 2 treatment cycles. However, plasma ctDNA analyses for this patient revealed weak T790M positivity, suggesting that a combination of chemotherapy and targeted third-generation EGFR-TKI treatment may have been more effective, although further research is necessary to fully determine the efficacy of this strategy. The other one of these 2 patients did not receive treatment, and succumbed to the disease due to rapid progression. At present, there is no standard treatment course for transformed SCLC. Given that this condition is serious and has a poor prognosis, it is crucial to develop novel treatments for affected patients $(43,44)$.

There were several limitations to the present study. First, our data were derived from electronic health records, which were not collected or organized with the goal of supporting research, and as such their accuracy and reliability are unknown. To compensate for this weakness, we have tried to collect original data, including radiographic images and gene detection reports where possible. In addition, as this was a single-center study of a small population, these results may not necessarily apply to larger populations. Furthermore, patients exhibiting gradual disease progression are more suitable candidates for re-biopsy owing to their overall good condition, but they are also able to continue using first-generation EGFR-TKIs. Although these patients exhibited T790M mutation positivity, they were not switched to osimertinib. Due to the relatively short follow-up period of the preent study, these patients were considered to have not changed their therapeutic regimen, although they may have done so after the end of our follow-up period.

In summary, the results of the present study suggest that NGS-based approaches are the most sensitive for detecting EGFR T790M mutations in plasma ctDNA samples from patients with NSCLC, with this approach yielding the highest concordance with tissue samples. In this study of a real-world clinical setting, it was also found that fewer patients than expected underwent re-biopsy, and that only a relatively limited subset of patients found to harbor the acquired T790M mutations underwent third-generation EGFT-TKI follow-up treatment. Furthermore, less than half of patients suffering from intracranial progression received osimertinib. Finally, it was also found that transformed SCLC with acquired T790M mutation positivity may be associated with poor prognosis.

\section{Acknowledgements}

Not applicable.

\section{Funding}

The present study was supported by the Wenzhou Municipal Science and Technology Bureau (grant. no. ZH2017001).

\section{Availability of data and materials}

The datasets generated and/or analyzed during the present study are available from the corresponding author on reasonable request.

\section{Authors' contributions}

TH, JZ and HX collected and organized the data, SS were responsible for formal analysis, JY proposed the methodology, and YL, TH and JZ wrote the manuscript. YL and TH revised the manuscript and approved the final version. All the authors have read and approved the final manuscript. TH and YL confirm the authenticity of the raw data.

\section{Ethics approval and consent to participate}

The present study was approved by the Institutional Review Board of the First Affiliated Hospital of Wenzhou Medical University (approval. no. 2017161), and in accordance with the 1964 Helsinki Declaration and its later amendments or comparable ethical standards.

\section{Patient consent for publication}

Not applicable.

\section{Competing interests}

The authors declare that they have no competing interests.

\section{References}

1. Bray F, Ferlay J, Soerjomataram I, Siegel RL, Torre LA and Jemal A: Global cancer statistics 2018: GLOBOCAN estimates of incidence and mortality worldwide for 36 cancers in 185 countries. CA Cancer J Clin 68: 394-424, 2018.

2. Wei WE, Mao NQ, Ning SF, Li JL, Liu HZ, Xie T, Zhong JH, Feng Y, Wei CH and Zhang LT: An analysis of EGFR mutations among 1506 cases of non-small cell lung cancer patients in Guangxi, China. PLoS One 11: e0168795, 2016.

3. Seo JS, Ju YS, Lee WC, Shin JY, Lee JK, Bleazard T, Lee J, Jung YJ, Kim JO, Shin JY, et al: The transcriptional landscape and mutational profile of lung adenocarcinoma. Genome Res 22: 2109-2119, 2012.

4. Mok TS, Wu YL, Thongprasert S, Yang CH, Chu DT, Saijo N, Sunpaweravong P, Han B, Margono B, Ichinose Y, et al: Gefitinib or carboplatin-paclitaxel in pulmonary adenocarcinoma. N Engl J Med 361: 947-957, 2009.

5. Rosell R, Carcereny E, Gervais R, Vergnenegre A, Massuti B, Felip E, Palmero R, Garcia-Gomez R, Pallares C, Sanchez JM, et al: Erlotinib versus standard chemotherapy as first-line treatment for European patients with advanced EGFR mutation-positive non-small-cell lung cancer (EURTAC): A multicentre, open-label, randomised phase 3 trial. Lancet Oncol 13: 239-246, 2012. 
6. Tan CS, Gilligan D and Pacey S: Treatment approaches for EGFR-inhibitor-resistant patients with non-small-cell lung cancer. Lancet Oncol 16: e447-e459, 2015.

7. Kobayashi S, Boggon TJ, Dayaram T, Jänne PA, Kocher O, Meyerson M, Johnson BE, Eck MJ, Tenen DG and Halmos B: EGFR mutation and resistance of non-small-cell lung cancer to gefitinib. N Engl J Med 356: 786-792, 2005.

8. Kosaka T, Yatabe Y, Endoh H, Yoshida K, Hida T, Tsuboi M, Tada H, Kuwano H and Mitsudomi T: Analysis of epidermal growth factor receptor gene mutation in patients with non-small cell lung cancer and acquired resistance to gefitinib. Clin Cancer Res 12: 5764-5770, 2006.

9. Camidge DR, Pao W and Sequist LV: Acquired resistance to TKIs in solid tumours: Learning from lung cancer. Nat Rev Clin Oncol 11: 473-481, 2014.

10. Sequist LV, Waltman BA, Dias-Santagata D, Digumarthy S, Turke AB, Fidias P, Bergethon K, Shaw AT, Gettinger S, Cosper AK, et al: Genotypic and histological evolution of lung cancers acquiring resistance to EGFR inhibitors. Sci Transl Med 3: 75ra26, 2011

11. Zhao J, Shao J, Zhao R, Li R, Yu K, Zhu L and Zhang J: Histological evolution from primary lung adenocarcinoma harboring EGFR mutation to high-grade neuroendocrine carcinoma. Thorac Cancer 9: 129-135, 2018.

12. Handorf EA, McElligott S, Vachani A, Langer CJ, Demeter MB, Armstrong K and Asch DA: Cost effectiveness of personalized therapy for first-line treatment of stage IV and recurrent incurable adenocarcinoma of the lung. J Oncol Pract 8: 267-274, 2012.

13. Kimura H, Kasahara K, Kawaishi M, Kunitoh H, Tamura T, Holloway B and Nishio K: Detection of epidermal growth factor receptor mutations in serum as a predictor of the response to gefitinib in patients with non-small-cell lung cancer. Clin Cancer Res 12: 3915-3921, 2006

14. Paweletz CP, Sacher AG, Raymond CK, Alden RS, O'Connell A, Mach SL, Kuang Y, Gandhi L, Kirschmeier P, English JM, et al: Bias-corrected targeted next-generation sequencing for rapid, multiplexed detection of actionable alterations in cell-free DNA from advanced lung cancer patients. Clin Cancer Res 22: 915-922, 2016.

15. Feng WN, Gu WQ, Zhao N, Pan YM, Luo W, Zhang H, Liang JM Yang $\mathrm{J}$ and Deng YM: Comparison of the superARMS and droplet digital PCR for detecting EGFR mutation in ctDNA from NSCLC patients. Transl Oncol 11: 542-545, 2018.

16. Li C, Jia R,Liu H,Zhang B and Wang C: EGFR T790M detection and osimertinib treatment response evaluation by liquid biopsy in lung adenocarcinoma patients with acquired resistance to first generation EGFR tyrosine kinase inhibitors. Diagn Pathol 13: 49, 2018.

17. Zhu G, Ye X, Dong Z, Lu YC, Sun Y, Liu Y, McCormack R, $\mathrm{Gu}$ Y and Liu X: Highly sensitive droplet digital PCR method for detection of EGFR-activating mutations in plasma cell-free DNA from patients with advanced non-small cell lung cancer. J Mol Diagn 17: 265-272, 2015

18. Vendrell JA, Mazieres J, Senal R, Rouquette I, Quantin X, Pujol JL, Roch B, Bouidioua A, Godreuil S and Coyaud E: Ultra-sensitive EGFR (T790M) detection as an independent prognostic marker for lung cancer patients harboring EGFR (del19) mutations and treated with first-generation TKIs. Clin Cancer Res 25: 4280-4289, 2019.

19. MokTS,Wu YL,AhnMJ,GarassinoMC,KimHR,RamalingamSS Shepherd FA, He Y, Akamatsu H, Theelen WS, et al: Osimertinib or platinum-pemetrexed in EGFR T790M-positive lung cancer. N Engl J Med 376: 629-640, 2017.

20. Seto T, Nogami N, Yamamoto N, Atagi S, Tashiro N, Yoshimura Y, Yabuki Y and Saka H: Real-World EGFR T790M Testing in advanced nonsmall-cell lung cancer: A prospective observational study in Japan. Oncol Ther 6: 203-215, 2018.

21. Jackman D, Pao W, Riely GJ, Engelman JA, Kris MG, Janne PA, Lynch T, Johnson BE and Miller VA: Clinical definition of acquired resistance to epidermal growth factor receptor tyrosine kinase inhibitors in non-small-cell lung cancer. J Clin Oncol 28: 357-360, 2010

22. Eisenhauer EA, Therasse P, Bogaerts J, Schwartz LH, Sargent D, Ford R, Dancey J, Arbuck S, Gwyther S, Mooney M, et al: New response evaluation criteria in solid tumours: Revised RECIST guideline (version 1.1). Eur J Cancer 45: 228-247, 2009.

23. Lindeman NI, Cagle PT, Aisner DL, Arcila ME, Beasley MB, Bernicker EH, Colasacco C, Dacic S, Hirsch FR, Kerr K, et al: Updated molecular testing guideline for the selection of lung cancer patients for treatment with targeted tyrosine kinase inhibitors Guideline from the college of American pathologists, the international association for the study of lung cancer, and the association for molecular pathology. Arch Pathol Lab Med 142: 321-346, 2018.
24. Wei Z, Shah N, Deng C, Xiao X, Zhong T and Li X: Circulating DNA addresses cancer monitoring in non small cell lung cancer patients for detection and capturing the dynamic changes of the disease. Springerplus 26: 531, 2016.

25. Diehl F, Schmidt K, Choti MA, Romans K, Goodman S, Li M, Thornton K, Agrawal N, Sokoll L, Szabo SA, et al: Circulating mutant DNA to assess tumor dynamics. Nat Med 14: 985-990, 2008.

26. Dawson SJ, Tsui DW, Murtaza M, Biggs H, Rueda OM, Chin SF, Dunning MJ, Gale D, Forshew T, Mahler-Araujo B, et al: Analysis of circulating tumor DNA to monitor metastatic breast cancer. N Engl J Med 368: 1199-1209, 2013.

27. Haber DA and Velculescu VE: Blood-based analyses of cancer: Circulating tumor cells and circulating tumor DNA. Cancer Discov 4: 650-661, 2014.

28. Usui K, Yokoyama T, Naka G, Ishida H, Kishi K, Uemura K, Ohashi $Y$ and Kunitoh H: Plasma ctDNA monitoring during epidermal growth factor receptor (EGFR)-tyrosine kinase inhibitor treatment in patients with EGFR-mutant non-small cell lung cancer (JP-CLEAR trial). Jpn J Clin Oncol 1: 554-558. 2019.

29. Zugazagoitia J, Gomez-Rueda A, Jantus-Lewintre E, Isla D, Camps C, Ramos I, Trigo JM, Bernabé R, Juan-Vidal O, Sanchez-Torres JM, et al: Clinical utility of plasma-based digital next-generation sequencing in oncogene-driven non-small-cell lung cancer patients with tyrosine kinase inhibitor resistance. Lung Cancer 134: 72-78, 2019.

30. Cui S, Ye L, Wang H, Chu T, Zhao Y, Gu A, Xiong L, Shi C and Jiang L: Use of superARMS EGFR mutation detection kit to detect EGFR in plasma cell-free DNA of patients with lung adenocarcinoma. Clin Lung Cancer 19: e313-e122, 2018.

31. Siravegna G, Marsoni S, Siena S and Bardelli A: Integrating liquid biopsies into the management of cancer. Nat Rev Clin Oncol 14: 531-548, 2017.

32. Diaz LA Jr and Bardelli A: Liquid biopsies: Genotyping circulating tumor DNA. J Clin Oncol 32: 579-586, 2014.

33. Nosaki K, Satouchi M, Kurata T, Yoshida T, Okamoto I, Katakami N, Imamura F, Tanaka K, Yamane $Y$, Yamamoto $\mathrm{N}$, et al: Re-biopsy status among non-small cell lung cancer patients in Japan: A retrospective study. Lung Cancer 101: $1-8,2016$

34. Arcila ME, Oxnard GR, Nafa K, Riely GJ, Solomon SB, Zakowski MF, Kris MG, Pao W, Miller VA and Ladanyi M: Rebiopsy of lung cancer patients with acquired resistance to EGFR inhibitors and enhanced detection of the T790M mutation using a locked nucleic acid-based assay. Clin Cancer Res 17: 1169-1180, 2011

35. Li Y, Xu Y, Wu X, He C, Liu Q and Wang F: Comprehensive analysis of EGFR T790M detection by ddPCR and ARMS-PCR and the effect of mutant abundance on the efficacy of osimertinib in NSCLC patients. J Thorac Dis 11: 3004-3014, 2019.

36. Oxnard GR, Thress KS, Alden RS, Lawrance R, Paweletz CP, Cantarini M, Yang JCH, Barrett JC, and Jänne PA: Association between plasma genotyping and outcomes of treatment with osimertinib (AZD9291) in advanced non-small-cell lung cancer. J Clin Oncol 34: 3375-3382, 2016.

37. Sakai K, Horiike A, Irwin DL, Kudo K, Fujita Y, Tanimoto A, Sakatani T, Saito R, Kaburaki K, Yanagitani N, et al: Detection of epidermal growth factor receptor T790M mutation in plasma DNA from patients refractory to epidermal growth factor receptor tyrosine kinase inhibitor. Cancer Sci 104: 1198-1204, 2013.

38. Wang Z, Chen R, Wang S, Zhong J, Wu M, Zhao J, Duan J, Zhuo M, An T, Wang Y, et al: Quantification and dynamic monitoring of EGFR T790M in plasma cell-free DNA by digital PCR for prognosis of EGFR-TKI treatment in advanced NSCLC. PLoS One 9: e110780, 2014

39. Wu YL, Cheng Y, Zhou X, Lee KH, Nakagawa K, Niho S, Tsuji F, Linke R, Rosell R, Corral J, et al: Dacomitinib versus gefitinib as first-line treatment for patients with EGFR-mutation-positive non-small-cell lung cancer (ARCHER 1050): A randomised, open-label, phase 3 trial. Lancet Oncol 18: 1454-1466, 2017.

40. Kuo CS, Huang CH, Liu CY, Pavlidis S, Ko HW, Chung FT, Lin TY, Wang CL, Guo YK and Yang CT: Prior EGFR-TKI treatment in EGFR-mutated NSCLC affects the allele frequency fraction of acquired T790M and the subsequent efficacy of osimertinib. Target Oncol 14: 433-440, 2019.

41. Soria JC, Ohe Y, Vansteenkiste J, Reungwetwattana T, Chewaskulyong B, Lee KH, Dechaphunkul A, Imamura F, Nogami N, Kurata T, et al: Osimertinib in untreated EGFRmutated advanced non-small-cell lung cancer. N Engl J Med 378: $113-125,2018$ 
42. Wang BX, Ou W, Mao XY, Liu Z, Wu HQ and Wang SY: Impacts of EGFR mutation and EGFR-TKIs on incidence of brain metastases in advanced non-squamous NSCLC. Clin Neurol Neurosurg 160: 96-100, 2017.

43. Heon S, Yeap BY, Lindeman NI, Joshi VA, Butaney M, Britt GJ Costa DB, Rabin MS, Jackman DM and Johnso BW: The impact of initial gefitinib or erlotinib versus chemotherapy on central nervous system progression in advanced non-small cell lung cancer with EGFR mutations. Clin Cancer Res 18: 4406-4414, 2012.

44. Porta R, Sanchez-Torres JM, Paz-Ares L, Massuti B, Reguart N, Mayo C, Lianes P, Queralt C, Guillem V, Salinas P, et al: Brain metastases from lung cancer responding toerlotinib: The importance of EGFR mutation. Eur Respir J 37: 624-631, 2011.
45. Niederst MJ, Sequist L, Poirier JT, Mermel CH, Lockerman EL, Garcia AR, Katayama R, Costa C, Ross KN, Moran T, et al: $\mathrm{RB}$ loss in resistant EGFR mutant lung adenocarcinomas that transform to small-cell lung cancer. Nat Commun 11: 6377, 2015. 46. Shiroyama T, Nasu S, Tanaka A, Takata S, Masuhiro K, Takada H, Morita S, Morishita N, Suzuki H, Okamoto N, et al: Transformation to small cell lung cancer after first-line afatinib treatment. Respir Med Case Rep 23: 188-190, 2018. 